\title{
Morphological variations of the peripapillary circle of Zinn-Haller by flat section
}

\author{
Myung-Kyoo Ko, Dong-Seob Kim, Yoon-Koo Ahn
}

\begin{abstract}
Aims-To evaluate the morphometric and morphological variations of the circle of Zinn-Haller (CZH) in the human eye.

Methods-42 human enucleated eyes were used in this study. After transverse flat thick sections were cut through the optic nerve and adjacent sclera, tissue sections were stained with haematoxylin and eosin or examined immediately by wet preparation under a light microscope. The average vessel diameter of the arterial circle and the average distance between the optic nerve head (ONH) and the arterial circle were determined. Various branching patterns of the $\mathrm{CZH}$ were also evaluated.

Results-The vessel diameter of the arterial circle was 123 (SD 75) $\mu \mathrm{m}$ (range 20-230 $\mathrm{\mu m})$. The distance of the CZH from the ONH margin was 403 (352) $\mu \mathrm{m}$ (0-1050 $\mu \mathrm{m})$. The CZH gave off branches to the optic nerve and to the peripapillary choroid (PPC) with various branching patterns especially at the entry point of paraoptic short posterior ciliary artery. Conclusions-The CZH exists within a variable distance from the $\mathrm{ONH}$ and its average diameter is similar to that of the central retinal vessels though it shows marked variation even in the same circle. The CZH also shows variable configurations in branching patterns. These variations may act as contributing factors that are responsible for the individual susceptibility of the anterior optic nerve and the PPC to circulatory disturbances.

(Br F Ophthalmol 1999;83:862-866)
\end{abstract}

The circle of Zinn-Haller $(\mathrm{CZH})$ is known to be an intrascleral arteriolar anastomosis derived from medial and lateral paraoptic short posterior ciliary arteries (SPCAs). The significance of this arterial circle in supplying the anterior optic nerve and peripapillary region has been the subject of controversy. ${ }^{1-11}$ However, based on continued reports using a microvascular corrosion casting of human eyes ${ }^{8-10} 1^{12-14}$ the concept of a major contribution of the $\mathrm{CZH}$ to these regions has gained wide acceptance.

There has been much clinical and experimental evidence supporting the vascular aetiology of the diverse spectrum of visual field defects and optic nerve head $(\mathrm{ONH})$ changes in high or normal tension glaucoma and in anterior ischaemic optic neuropathy (AION).$^{815-20}$ However, their exact pathogenesis has remained unclear because of the difficulty in ascertaining the complete anatomical and physiological system of blood flow of the $\mathrm{ONH}$ and the peripapillary choroid (PPC). Knowledge of the detailed microvascular anatomy in these regions is essential to understand the possible role of the vascular system in diseases of the optic nerve.

Among the various examination methods used to observe the $\mathrm{CZH}$, a conventional serial histological section method was considered to be difficult to interpret, particularly when the vessels lie in different planes and have small diameters. ${ }^{5}{ }^{14}$ In the present study, we used the flat thick section technique to obtain a considerable proportion of the $\mathrm{CZH}$ in one tissue section, and evaluated its morphology by several factors - vessel diameter, distance from the $\mathrm{ONH}$ margin, and branching patterns.

\section{Materials and methods}

This research protocol followed the tenets of the Declaration of Helsinki. We studied 42 enucleated human eyes from 42 subjects. They were collected from the eye clinic of Hanyang University Hospital from March 1976 until December 1994. Of the 42 subjects, 22 were male and 20 were female. Ages ranged from 20 to 63 years, with a mean of 46.5 years. The enucleated eyes were from a ruptured eyeball (15 eyes), from phthisis bulbi (14 eyes), from intraocular malignancy (five eyes), from absolute glaucoma (five eyes), and from donor sources (three eyes). After being divided into half, the eyeballs were turned inside out to expose the retinal surface, and then the vitreous, retina, and choroid were removed. Transverse lamellar flat thick sections (three sections within the intrascleral portion of the optic nerve) were made by razor blade, beginning at about $2 \mathrm{~mm}$ from the optic disc margin to involve the optic nerve as well as the adjacent sclera (Fig 1). The sections were stained with haematoxylin and eosin or examined immediately by wet preparation under a light microscope.

We measured the vessel diameter of the $\mathrm{CZH}$, which was defined as an interval between the outer margins of the vessel wall, with a calibrated eye piece graticule. Using the

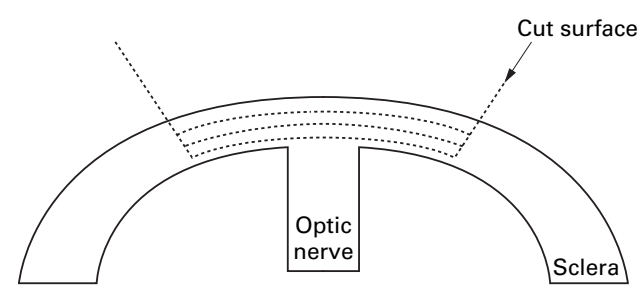

Figure 1 Schematic diagram of flat section method. The cut surface (dotted line) was made along the optic nerve and adjacent sclera with the eyecup everted.
Accepted for publication 1 March 1999 


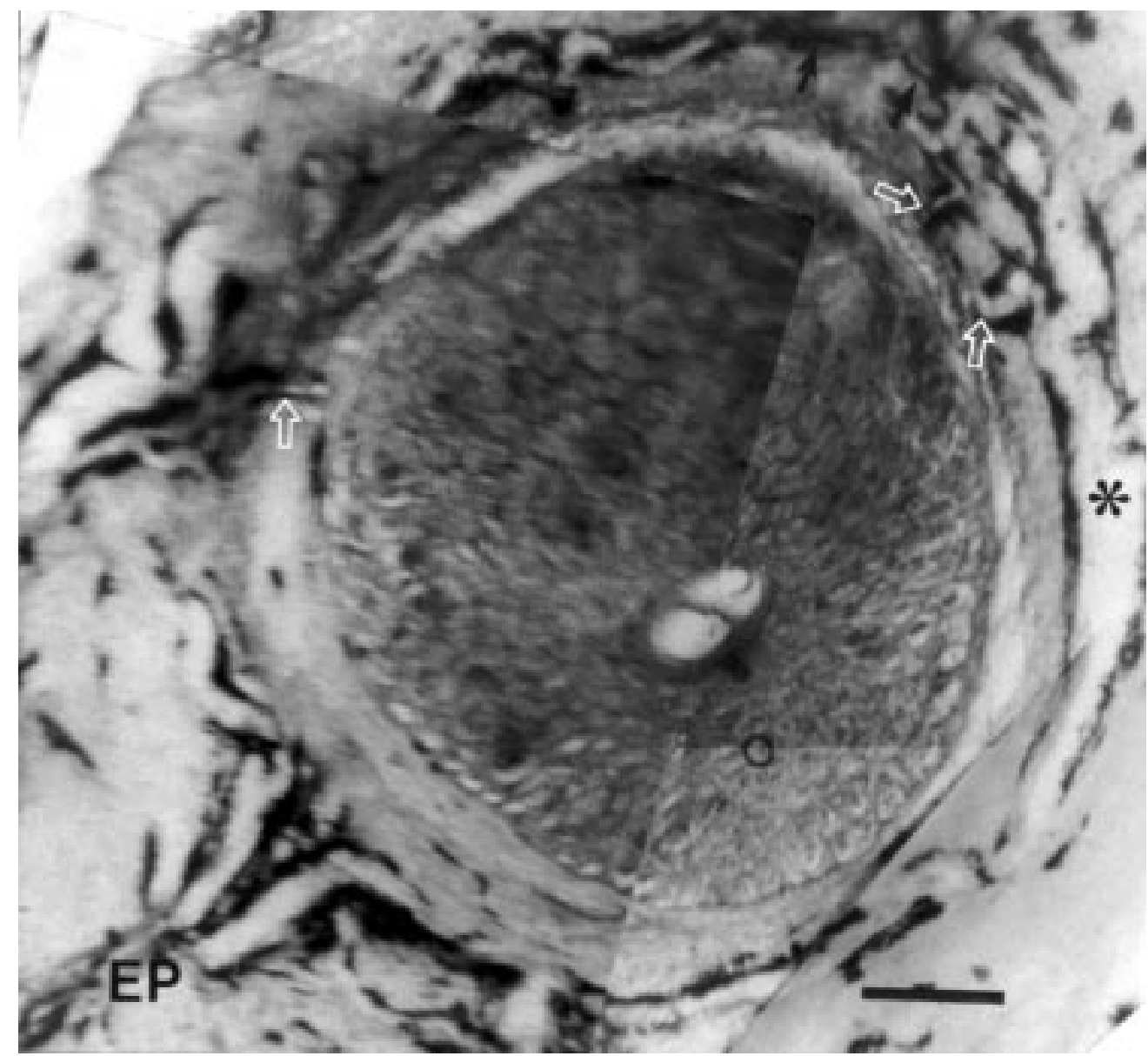

Figure 2 Light micrograph of flat sections of the optic nerve and adjacent sclera from a 32 year old male patient with right eyeball rupture showing the complete arterial circle of Zinn-Haller around the optic nerve (haematoxylin and eosin). Even in the same arterial circle the vessel diameter shows marked fluctuation, so the minimum value is about $20 \mu \mathrm{m}$ (black arrows) and the maximum is $230 \mu \mathrm{m}$ (asterisk). Note the entry point (EP) of the paraoptic short posterior ciliary artery, several branches to the optic nerve (white arrows) from the main arterial circle and sudden decrease of the intraluminal. diameter (narrow portion, between black arrows) in the main body of the arterial circle. The bar represents $400 \mu \mathrm{m}$.

same method we measured the distance between the point of the optic nerve margin and the point of the inner wall of the arterial circle. We calculated the mean values, and obtained the average vessel diameter and the average distance from the mean values of all specimens. Additionally, the variable branching patterns at the entry points of paraoptic SPCAs and from the main bodies of the vascular circle were also noted.

\section{Results}

The main vessel diameter of the $\mathrm{CZH}$ was 123 (SD 75) $\mu \mathrm{m}$ (range 20-230 $\mu \mathrm{m}$ ) (Fig 2) but showed marked variation between subjects and even in the same subject. The variation in diameter in the same subject means that the circle did not have a uniform intraluminal diameter in its course; furthermore, in some cases the calibre of the vessels suddenly became narrower (Fig 2).

The mean distance of the $\mathrm{CZH}$ from the $\mathrm{ONH}$ margin was 403 (SD 352) $\mu \mathrm{m}$. It was mainly influenced by the location of the entry point of the paraoptic SPCAs and the concentricity of the main body of the CZH. The maximum and the minimum values were 1050 $\mu \mathrm{m}$ (Fig 3) and zero (Fig 4A) respectively, rep- resenting marked individual variation relative to the optic nerve.

Various branching patterns of the $\mathrm{CZH}$ (Fig 5) especially at the entry of the paraoptic SPCA into sclera were noted-namely, asymmetric bifurcation of paraoptic SPCA with one branch having different intraluminal diameters (Fig 4B), bifurcate (Fig 4A, C), and trifurcate (Fig 4D) divisions arising from one vascular trunk of the paraoptic SPCAs. These divisions constituted vascular trunks, which might become a main body of the circle, a branch to the optic nerve, and a branch to the choroid. We could also observe the minor circle (Figs 3, 5), the anastomosing arterioles (Figs 3, 5), and several branches to the optic nerve (Fig 2) and to the PPC (Figs 4C, 5) from the main arterial circle.

\section{Discussion}

In this study we employed a method of transverse flat thick sections of the intrascleral portion of optic nerve with adjacent sclera to observe the microvascular anatomy of the $\mathrm{CZH}$. The advantage of using flat thick sections is the ability to obtain a large proportion of the $\mathrm{CZH}$, which exists at variable distances from the $\mathrm{ONH}$, in one tissue section. 


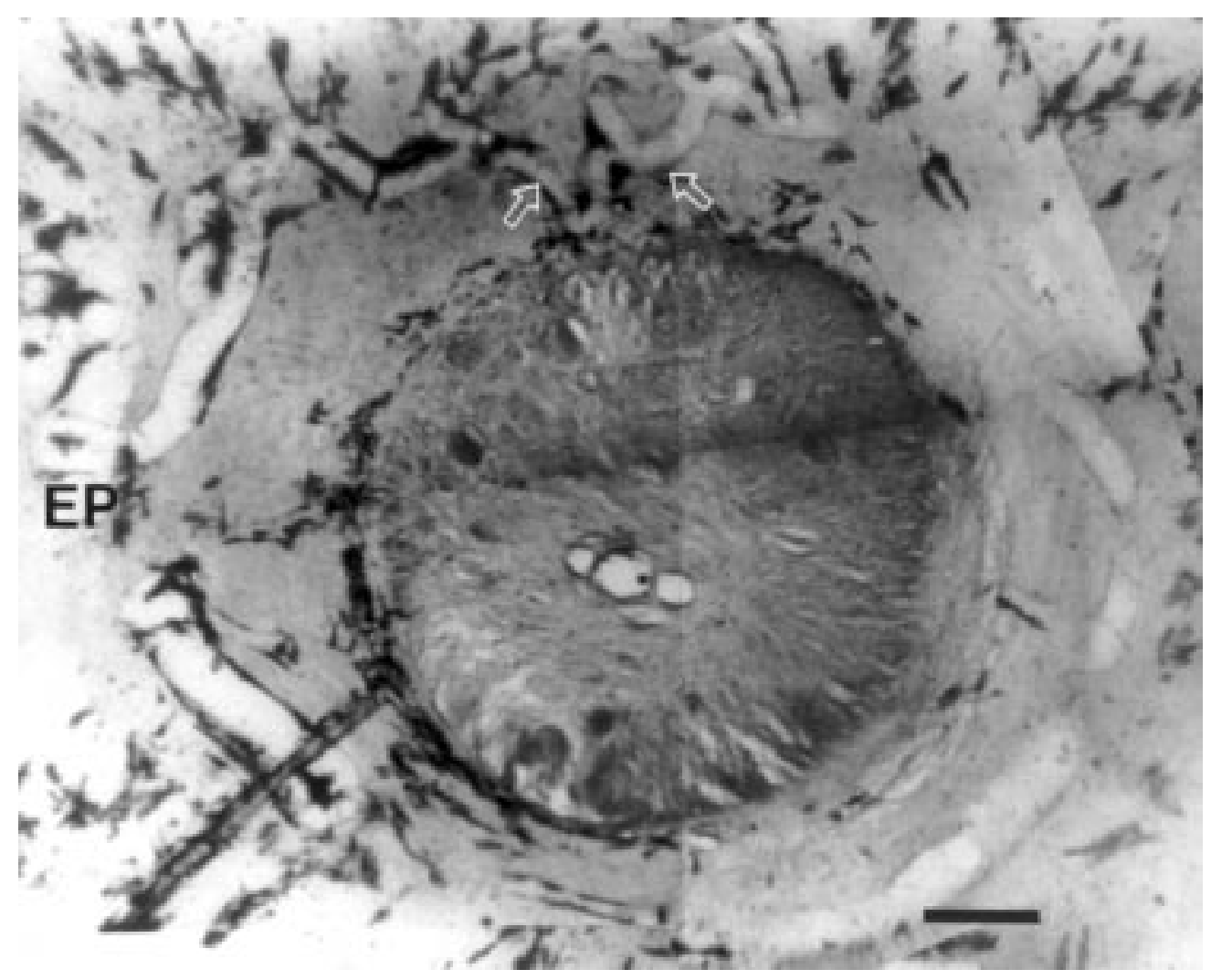

Figure 3 Light micrograph of flat sections of the optic nerve and adjacent sclera from a 61 year old female patient with absolute glaucoma showing the arterial circle of Zinn-Haller around the optic nerve (haematoxylin and eosin). The entry point (EP) of the paraoptic short posterior ciliary artery is far away from the optic nerve margin. Note the minor circle (arrows) with anastomosing arteriole. The bar represents $400 \mu \mathrm{m}$.

Moreover, turning the eyeball inside out before preparing the transverse section made it easy to cut the specimens parallel to the curvature of the eyeball.

Using the microvascular corrosion casting technique with human eyes many investigators $^{8-10}$ 12-14 have described the three dimensional angioarchitecture of the $\mathrm{ONH}$, and the $\mathrm{CZH}$ is considered to be a consistent vessel in humans and to have a crucial role in supplying the anterior optic nerve and the PPC. Olver and associates, ${ }^{8}{ }^{14}$ using this casting method, have already documented the morphological variations of the $\mathrm{CZH}$ in terms of form, position, and branches. However, we considered flat section method to be suitable for the present study in which the variations of the $\mathrm{CZH}$ could be measured relative to the position of surrounding structures.

In our previous study ${ }^{21}$ we have reported the variations of the $\mathrm{CZH}$ in location, shape, and the appearance of the entry point of the temporal paraoptic SPCA. Similarly, as shown in the present study, the location of the entry point of the paraoptic SPCA and the main body of the $\mathrm{CZH}$ showed considerable individual variation. So they could lie at a distance from (Fig 3) or in the vicinity of (Fig 4A) the optic nerve. The vessel diameter of the $\mathrm{CZH}$ also showed individual variation and its average $(123 \mu \mathrm{m})$ was similar to that of central retinal vessels. Even in the same individual the circle diameter showed fluctuation in its course. Of special note was the abrupt decrease in the vessel calibre, the narrow portion, which was defi- nitely a part of the main body of the continuing circle. It could be observed at the entry point of the paraoptic SPCA (Fig 4B) or in the middle of the course of the $\mathrm{CZH}$ (Fig 2).

Branches originating from the $\mathrm{CZH}$ have already been described in previous reports, , $^{-10} 14$ and they are branches to the optic nerve (prelaminar and laminar portion), to the pial arterial system, and to the PPC. However, our results showed the complex branching pattern of the CZH especially at the entry point of the paraoptic SPCA - asymmetric bifurcation from the same paraoptic SPCA, and bifurcate or trifurcate divisions from one vascular trunk of the paraoptic SPCAs. In addition, we also observed a minor circle within the arterial circle and numerous anastomosing arterioles (Figs 3, 5) between branches and between the main body of the circle and its branches in some of the CZH. The presence of a minor circle or many anastomosing arterioles may imply the presence of a collateral circulation.

All the morphological variations shown in the present study may act as possible contributing factors which influence the individual susceptibility of the ONH and the PPC to circulatory disturbances. Hence, the proximity of the $\mathrm{CZH}$ to the $\mathrm{ONH}$, the absence of the narrow portion, the absence of asymmetric bifurcation, and the presence of many anastomosing arterioles may construct an anatomical protective system in the vascular circle providing resistance against potential circulatory disturbance or ischaemic insult. 

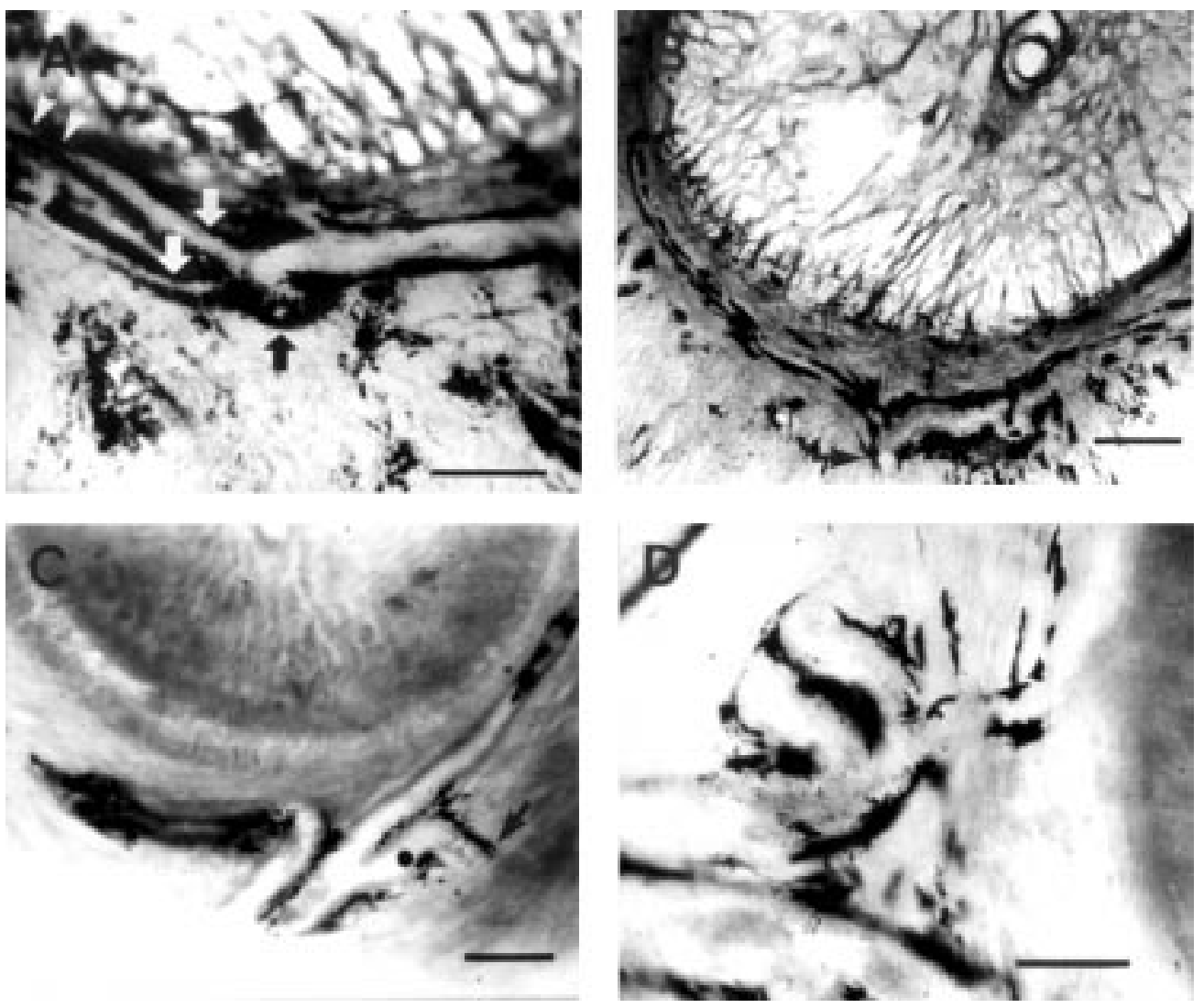

Figure 4 Light micrographs of flat sections of the optic nerve and adjacent sclera showing the variation of the circle of Zinn-Haller (CZH) at the entry point of the paraoptic short posterior ciliary artery (PSPCA). (A) The CZH, in some part, adjoins the optic nerve margin (white arrowheads), and the distance from the optic nerve margin to the inner wall of the arterial circle is nearly zero. Note the entry point (black arrows) of the PSPCA in the vicinity of the optic nerve margin and bifurcate divisions (white arrows) from one vascular trunk of the PSPCA. (B) The CZH runs in the opposite direction with different intraluminal diameter (asymmetric bifurcation) from the entry point (arrow) of PSPCA. (C) The CZH has bifurcate divisions at the entry point of the PSPCA, one of which go to the peripapillary choroid (arrow). (D) The CZH has trifurcate divisions at the entry point of the PSPCA. (Haematoxylin and eosin $(A, B)$, wet preparation $(C, D)$.) The bar represents $200 \mu \mathrm{m}(A, D)$ and $400 \mu \mathrm{m}(B, C)$.

We cannot account for any age related or other unknown factors influencing the results of our study, and we cannot be sure whether the vascular anatomy seen in advanced pathological conditions is relevant to the normal pattern of the $\mathrm{CZH}$. In this regard, we recognised the limitations of our study. However, we believe that our results would be useful in studying the circulation of the $\mathrm{ONH}$ and PPC, and showing that the CZH has variable morphology and its anatomical features may be related to the difference in the individual susceptibility of the optic nerve and PPC to circulatory disturbances. Hence, the variable visual outcomes associated with non-arteritic AION,${ }^{1722-24}$ and even with glaucoma and

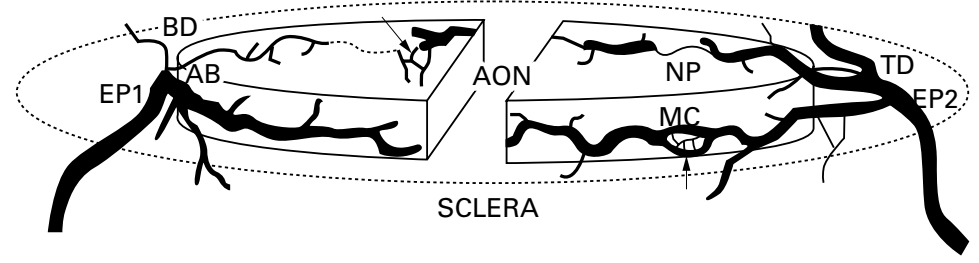

Figure 5 Schematic diagram showing the major variations of the circle of Zinn-Haller. $A O N=$ anterior optic nerve, $E P 1=$ entry point of the paraoptic short posterior ciliary artery (PSPCA) adjacent to the optic nerve; EP2 = entry point of the PSPCA away from the optic nerve; $B D=$ bifurcate divisions; $T D=$ trifurcate divisions; $A B=$ asymmetric bifurcation; $N P=$ narrow portion; $M C=$ minor circle with anastomosing arterioles (arrows). Dotted line represents a part of the arterial circle that may be in the extrascleral region. allied disorders may be attributed to the location of ischaemia in the $\mathrm{CZH}$ and to the variable morphology of the $\mathrm{CZH}$ with or without a protective system. Further investigation will be needed to clarify the complete nature of the anatomical and physiological system of the ONH blood flow in the normal and pathological eyes in the future.

This work was presented in part at the Annual Meeting of the Association for Research in Vision and Ophthalmology (ARVO), May 1997, in Fort Lauderdale, Florida, USA.

1 Henkind P, Levitzky M. Angioarchitecture of the optic nerve, I: the papilla. Am f Ophthalmol 1969;68:979-86.

2 Levitzky M, Henkind P. Angioarchitecture of the optic nerve, II: lamina cribrosa. Am f Ophthalmol 1969;68:98696.

3 Anderson DR. Vascular supply to the optic nerve of primates. Am f Ophthalmol 1970;70:341-51.

4 Anderson DR, Braverman, S. Reevaluation of the optic disc vasculature. Am f Ophthalmol 1976;82:165-74.

5 Leiberman MF, Maumenee AE, Green WR. Histologic studies of the vasculature of the anterior optic nerve. Am $\mathcal{F}$ Ophthalmol 1976;82:405-23.

6 Hayreh SS. Blood supply and vascular disorders of the optic nerve. An Inst Barraquer 1963;4:7-109.

7 Hayreh SS. Blood supply of the optic nerve head and its role in optic atrophy, glaucoma, and oedema of the optic nerve. Brf Ophthalmol 1969;53:721-48.

8 Olver JM, Spalton DJ, McCartney ACE. Microvascular study of the retrolaminar optic nerve in man: the possible significance in anterior ischemic optic neuropathy. Eye 1990;4:7-24.

9 Zhao Y, Li F. Microangioarchitecture of optic papilla. $f p n \mathcal{F}$ Ophthalmol 1987;31:147-59.

10 Onda E, Cioffi GA, Bacon DR, et al. Microvasculature of the human optic nerve. Am f Ophthalmol 1995;120:92-102. 
11 Hayreh SS. Anatomy and physiology of the optic nerve head. Trans Am Acad Ophthalmol Otolaryngol 1974;78:24054 .

12 Fryczkowski AW, Grimson BS, Peiffer RL Jr. Scanning electron microscopy of vascular casts of the human scleral lamina cribrosa. Int Ophthalmol 1984;7:95-100

13 Olver JM. Functional anatomy of the choroidal circulation: methyl methacrylate casting of human choroid. Eye 1990;4:262-72

14 Olver JM, Spalton DJ, McCartney ACE. Quantitative morphology of human retrolaminar optic nerve vasculature. Invest Ophthalmol Vis Sci 1994;35:3858-66.

15 Hayreh SS, Revie HIS, Edwards J. Vasogenic origin of visual field defects and optic nerve changes in glaucoma. $\mathrm{Br} f$ Ophthalmol 1970;54:461-72.

16 Hayreh SS. Inter-individual variation in blood supply of the optic nerve head: its importance in various ischemic disorglaucoma and allied disorders. Doc Ophthalmol 1985;59: glaucoma

17 Hayreh SS. Anterior ischemic optic neuropathy. Arch Neurol 1981;38:675-8.
18 Francois J, Neetens A. Vascularity of the eye and the optic nerve in glaucoma. Arch Ophthalmol 1964;71:219-25.

19 Spaeth GL. Pathogenesis of visual loss in patients with glaucoma. Trans Am Acad Ophthalmol Otolaryngol 1971;75:

20 Rader J, Feuer WJ, Anderson DR. Peripapillary vasoconstriction in the glaucomas and the anterior ischemic optic neuropathies. Am f Ophthalmol 1994;117:72-80.

$21 \mathrm{Ko}$ MK, Kim DS, Ahn YK. Peripapillary circle of Zinn-Haller revealed by fundus fluorescein angiography. $\mathrm{Br}$ f Ophthalmol 1997;81:663-7.

22 Repka MX, Savino PJ, Schatz NJ, et al. Clinical profile and long-term implications of anterior ischemic optic neuropathy. Am f Ophthalmol 1983;96:478-83.

23 Boone MI, Massry GG, Frankel RA, et al. Visual outcome in bilateral nonarteritic anterior ischemic optic neuropathy. Ophthalmology 1996;103:1223-8.

24 Ischemic Optic Neuropathy Decompression Trial Study Group. Characteristics of patients with nonarteritic anterior ischemic optic neuropathy eligible for the ischemic optic neuropathy decompression trial. Arch Ophthalmol 1996;114:1366-74. 